\title{
Cognitive closed access femtocell application using multi-element antenna
}

\author{
Mohammad Tariqul Islam*, Afaz Uddin Ahmed, Mandeep Singh Jit Singh, Mahamod Ismail, Tharek Bin Abd Rahman \\ and Norbahiah Misran
}

\begin{abstract}
In this paper, a cognitive closed access multi-element antenna-specific femtocell protocol is presented. Femtocell is considered as the preeminent solution for indoor coverage in long-term evaluation (LTE) and LTE-advanced networks. The protocol is verified using a patch antenna for LTE network. In a vast deployment of this mini base station, unwanted handover event is considered as the major obstacle. Access control mechanism with coverage-optimized antenna pattern is one of the most promising solution to resolve this obstacle. A previously proposed microstrip four-element femtocell configuration is used to analyze the performance in user categorizing technique for closed access femtocell network. The categorization is performed using multi-layer feed forward network in neural network. The performance of the technique shows an inherent relationship between completeness of training data and error percentage. Sufficiently low error rates can be achieved by presenting about 15 training samples.
\end{abstract}

Keywords: Multi-element antenna; Femtocell; Closed access control; Neural network

\section{Introduction}

Femtocell is a low-power and low-cost data access point that provides high-quality coverage with voice and data services. It communicates with the core network through a wired broadband connection such as cable modem or digital customer line (DSL). The femtocell has a broad prospect in offices and residences as it can handle higher data traffic with less resources allocated than the macrocell. It extends the macrocell coverage at the cell edge regions. However, in dense femtocell network, under existing macrocell coverage, interference and unwanted handover events cause complicacy. In areas with multiple femtocell coverage, signaling congestion increases along with the incessant handover and handoff events. Therefore, disparate solution is required to avoid unwanted handover and interference due to overlapping of coverage. In indoor environment, the performance optimization of the femtocell is mainly dependent on its relative position and allocated resources. The femtocell is usually placed in corners of any residence or apartment, where it is easy to plug in the internet cable (DSL) and the power port. Conventional

\footnotetext{
* Correspondence: titareq@gmail.com

Department of Electrical, Electronic and Systems Engineering, University Kebangsaan Malaysia, Jalan Reko, 43600 Bangi, Selangor, Malaysia
}

single-element omnidirectional antenna is less suitable for femtocell coverage optimization as it radiates in all direction at the same intensity. Using multi-element microstrip patch, the antenna mounted on the square/ round surface of the femtocell device can be more effective to reduce coverage overlapping.

Access control mechanism has a vast effect on interference level in femtocell network. It can classify the users' access and the level of service. The mechanism of accesscontrolled femtocell depends on mainly on the mode selection [1,2]. Three access control modes are used in the femtocell network: open access, closed access, and hybrid access. Selection of each mode enables different mechanisms to serve network users.

Open access allows all the users in the network to connect within the coverage range. Closed access only allows particular users into the femtocell service with less sharing of bandwidth. This particular group is named closed subscriber group (CSG). There is another mode called hybrid access where a limited femtocell service are available for all the users but only the CSG are allowed to use the highest service. In a dense network, open access is a reason for increased number of handover and mobility events. It also shares bandwidth that might decrease the satisfaction level of femtocell owners. It increases 
the capacity of the network, thus sacrificing network stability. Closed access thus avoids frequent handover and mobility events, but under its superior coverage, it continuously influences the unwanted user to send out handover requests. This induces a new set of cross-tier interference. Hybrid access deals with both problem by adjusting resources according to the number of femtocell owners and subscribers [3,4]. However, for different density of users and femtocell locations, most of the algorithms are outperformed in practical implementation. Then again, owners buy femtocell for their personal benefits. They do not like to share the bandwidth with the random number of user unless they are within their residence.

In closed access, the users are pre-defined and the access of users is filtered out in the core layer. However, in the physical layer, the femtocell might overshoot the non-subscribers, as the femtocells are not configured according to its position in the residence. The use of multi-element antenna configuration for femtocell application was proposed in some literatures. A switch-based multi-element antenna configuration is described in [5], where switching between the antennas changes the radiation pattern of the femtocell. Another multi-element antenna structure was described with tunable attenuator in [6]. Multi-element antenna gives distinct gain patterns in different directions for each element. Using the variation of the gain patterns, antennas can be used to differentiate between the outdoor users and indoor users. By using neural network, the femtocell can be trained to perform as a closed access femtocell network by utilizing the receiving gain of the antennas. Figure 1 shows the concept of multi-element antenna configuration in closed access femtocell network.

Studies regarding smart antenna modeling, adaptive beam forming, and DOA estimation are based on the neural network solution [7-14]. Access control mechanism in femtocell is an application of MAC layer. In response to a random user request, it contends the entire signaling process to core network to reject the user up until it is under the femtocell coverage. Restricting the user from the femtocell end with a user categorizing technique will decrease the signaling congestion. In this paper, a neural network-based closed access femtocell network is proposed by using the variations of receiving gains of multi-element femtocell. A previously designed LTE multi-band microstrip antenna [15] is used for modeling the multi-element femtocell configuration. Results show that the femtocell categorizes the users without any mistake after a certain number of training samples. The rest of the paper is arranged as follows: antenna specification in Section 2, cognitive closed access femtocell in Section 3, simulation and results in Section 4, and conclusions in Section 5.

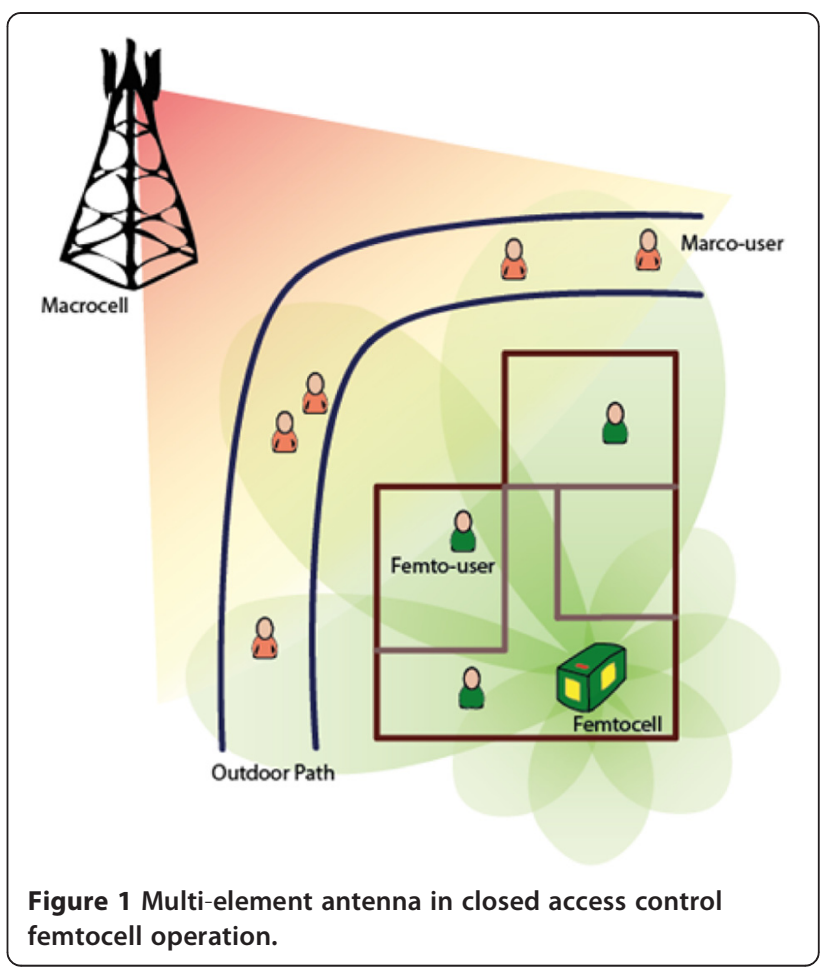

\section{Antenna specification}

The femtocell device considered here consists of fourelement microscript antennas with the design specification given in [16]. The antenna is designed using substrate material FR4 with relative permittivity of 4.6 , dielectric losstangent of 0.02 and thickness of $1.6 \mathrm{~mm}$. The antenna covers band 7 of LTE with a bandwidth of $230 \mathrm{MHz}$ starting from 2.48 to $2.71 \mathrm{GHz}$. The antennas are mounted on the femtocell surface facing $90^{\circ}$ apart from each other on the same axis like shown in Figure 2. The antenna specifications are done using CST Studio Suite. Figure 3a illustrates the far field radiation characteristics of the antenna at $2.6 \mathrm{GHz}$. The main lobe direction is around $0^{\circ}$. The maximum gain at $2.6 \mathrm{GHz}$ is $5.2 \mathrm{~dB}$. The far field radiation pattern of all the elements is shown in Figure 3b.

\section{Cognitive closed access femtocell}

The power density received by an antenna varies with the incidence angle. While receiving the signal, the antenna responds to an incoming wave from a given direction according to the pattern value in that direction. Each of $M$ number of antenna elements holds different gain patterns in each direction. Therefore, the received power at each element from a particular angle varies due to the prospective antenna gain. Using the variation of received power for the $M$ elements, the femtocell is trained to distinguish the outdoor and indoor users. The conversion of the power from user equipment to the antenna end also depends on the path loss, user equipment class, transmitting antenna gain, multi- 


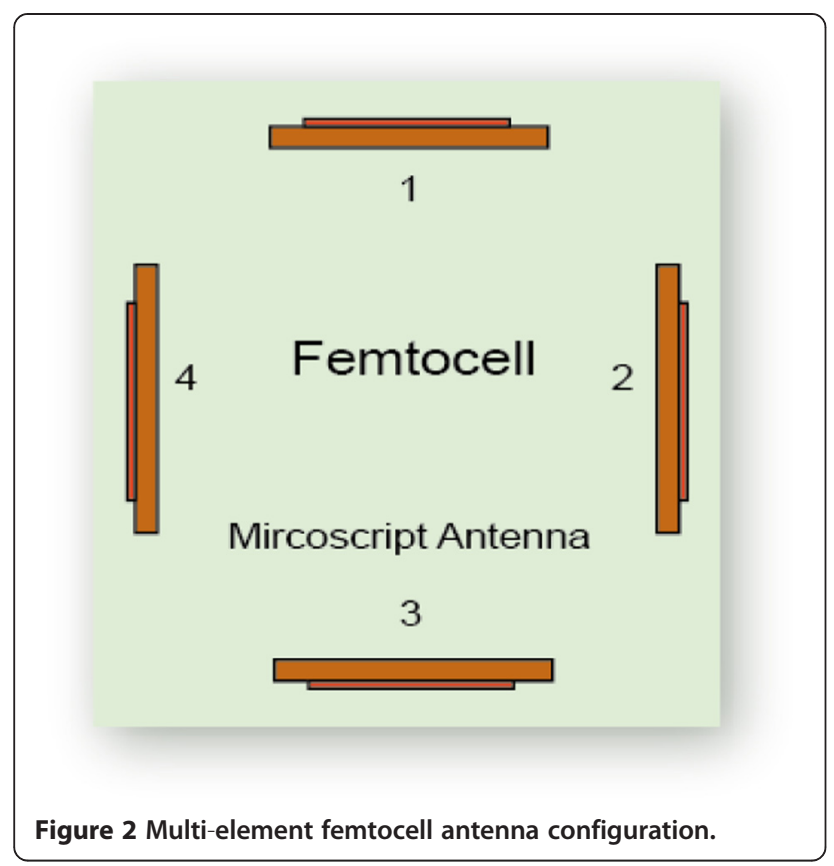

path fading, shadowing, noise signals, and receiver port and feed cable losses. However, other terms vary very little within the elements as in practical; femtocells are small in size, with antenna placed very close to each other. The user antennas are considered isotropic that can transmit in all directions evenly. Even if the antenna has directional radiation pattern, it will obtain the $M$ antenna elements location within a small angle from the user end.

Let us consider that the number of source is $K$. For $K$ th source, the incident beam plane wave centered at frequency $w$ impinging on the $M$ elements of antenna in different angle $\theta_{K}$. The maximum received power on the effective area of the antenna relates proportionally to the transmitting power of the user equipment. Using complex power presentation, the received power at $i$ th antenna element can be written as:

$$
P_{i}(t)=\sum_{m=1}^{K} Q_{m}(t) e^{-j k_{m i}}+n_{i}(t) ; i=1,2, \ldots M
$$

where,

$$
k_{m i}=k_{0} \sqrt{x_{i}^{2}+y_{i}^{2}} \operatorname{Cos}\left(\tan ^{-1} \frac{y_{i}}{x_{i}}\right)
$$

The center point of the femtocell is $(0,0)$ and coordinates of each antenna element center point is $\left(x_{i}, y_{i}\right)$. Using vector notation and Friss transmission law, Equation 1 can be expressed as:

$$
\begin{aligned}
& P(t)=A Q(t)+N(t) \\
& P(t)=\left[P_{1}(t), P_{2}(t) \ldots P_{M}(t)\right]^{T} \\
& Q(t)=\left[Q_{1}(t), Q_{2}(t) \ldots Q_{K}(t)\right]^{T} \\
& N(t)=\left[n_{1}(t), n_{2}(t) \ldots n_{M}(t)\right]^{T} \\
& A=\left(\frac{\lambda_{w} G_{R}\left(\theta_{1}\right)}{4 \pi R_{1}}, \frac{\lambda_{w} G_{R}\left(\theta_{2}\right)}{4 \pi R_{2}}, \ldots \frac{\lambda_{w} G_{R}\left(\theta_{K}\right)}{4 \pi R_{K}}\right)
\end{aligned}
$$

The noise signal is independent of $Q(t)$ for all antenna elements. The spiral co-relation matrix of the received power is as follows:

$$
\begin{aligned}
R & =E\left\{P(t) P(t)^{H}\right\} \\
& =A E\left[Q(t) Q^{H}(t)\right] A^{H}+E\left[N(t) N^{H}(t)\right]
\end{aligned}
$$

Here, $H$ is the conjugate transpose. The femtocell is trained to perform mapping of $G: R^{K} \rightarrow C^{M}$ from the space of $Q(t)$ to the $P(t)$. The neural network is used to do the inverse mapping $F: C^{M} \rightarrow R^{K}$.

In sample data, processing algorithm for signals applies the correlation matrix instead of actual output value. Here, the $Z$ is formed from the first row of the correlation matrix $R$ shown in N-MUST algorithm [14].
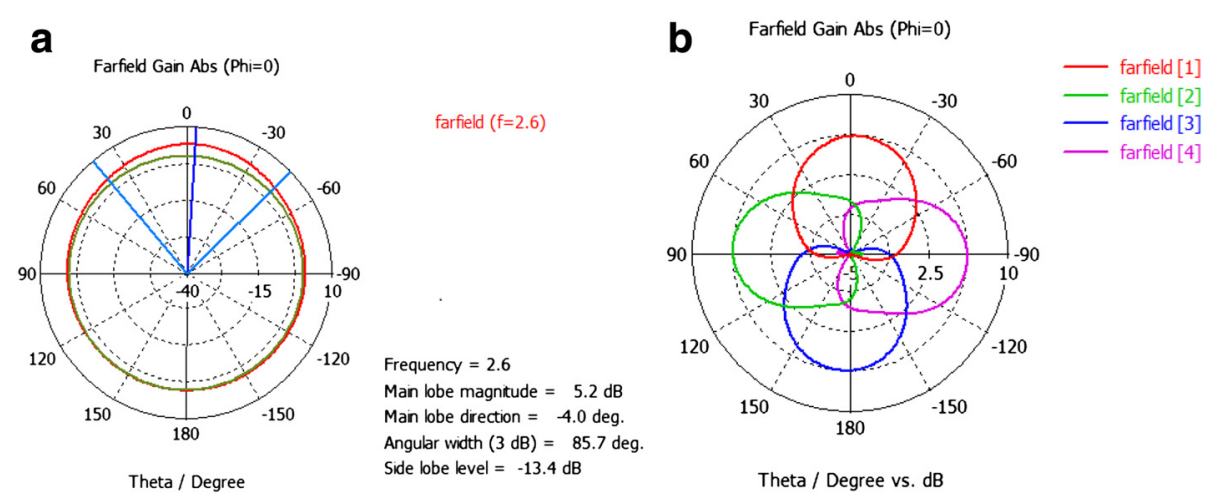

Figure 3 Radiation pattern at frequency $2.6 \mathrm{GHz}(\mathrm{a})$ and far field radiation patter of all the elements (b). 


$$
\begin{aligned}
& R=\left[\begin{array}{c}
R_{1,1} \ldots \ldots \ldots R_{1, M} \\
\ldots \ldots \ldots \ldots \ldots \ldots . \\
R_{M, 1} \ldots \ldots \ldots R_{M, M}
\end{array}\right] \\
& Z=\frac{b}{|b|}
\end{aligned}
$$

The size of $b$ is $M \times 1$, where $b$ got both real and imaginary values. Since the neural network does not deal with the imaginary value, the size of $Z$ is $2 M \times 1$. However, in [14], the authors calculated the angle of incidence only. The angle of incidence and the received power can be utilized (in theory) to train a neural network to identify the relative location of a user and grant access accordingly. This process appears rather redundant as the whole process can be carried out by a single neural network. Thus, in this paper, a neural network is trained using the vectors of comprising of $E$ from all antennas over multiple instances of $n$.

$$
\begin{aligned}
& E_{i, n}=\int_{n \times T}^{T(n+1)} P_{i}(t) d t \\
& \bar{E}=\left[\begin{array}{c}
E_{1, n} \\
\cdot \\
\cdot \\
E_{i, n}
\end{array}\right]
\end{aligned}
$$

where, $n=0,1,2,3 \ldots$ and $T$ is the sampling period.

The architecture of this paper involves two stages. The initial stage is the training process, that is to learn the traits of indoor and outdoor user using the value of $\bar{E}$. In the next stage, the network will detect the user based on the previous learning. The network will be trained initially with some sample of indoor and outdoor user's $\bar{E}$. After the training, random users are generated. They are
Table 1 System parameters for simulation

\begin{tabular}{ll}
\hline System parameters & Value/range \\
\hline Indoor samples & 30,300 \\
Outdoor samples & 30,200 \\
Randomly generated users (after training) & 30 \\
Femtocell antenna height & $1 \mathrm{~m}$ \\
User equipment height & $1 \mathrm{~m}$ \\
Frequency & $2.6 \mathrm{GHz}$ \\
UE transmit power (fixed) & $13 \mathrm{dBm}$ \\
Indoor wall loss & $5 \mathrm{~dB}$ \\
Outdoor wall loss & $8 \mathrm{~dB}$ \\
Shadow fading std & $6 \mathrm{~dB}$ \\
White noise power density & $-174 \mathrm{dBm} / \mathrm{Hz}$ \\
\hline
\end{tabular}

considered as isotropic sources. The values of $\bar{E}$ are calculated using Hata path loss model and considering unit shadow-fading and additive white Gaussian noise. When a source radiates towards the femtocell antenna elements, the varying gain of each antenna creates discrepancies in the received power. The neural network exploits these discrepancies to predict the category of the source. The neural network is trained to grant access if the location of the user is inside a certain region [17]. Based on the training experiences, the femtocell distinguishes the indoor and outdoor users. For random values of $\bar{E}$, the neural network will determine the users' category by giving an output of ' 1 ' or ' -1 ' (sigmoid output neurons). A filtering stage is shown in MN-MUST algorithm that reduces the size of the correlation matrix for cylindrical array configuration for direction of arrival estimation [7]. In this case, the received power is a function of the antenna gain in the impinging direction. For a single user, the more power pattern in each antenna element is considered, the more perfection is achieved

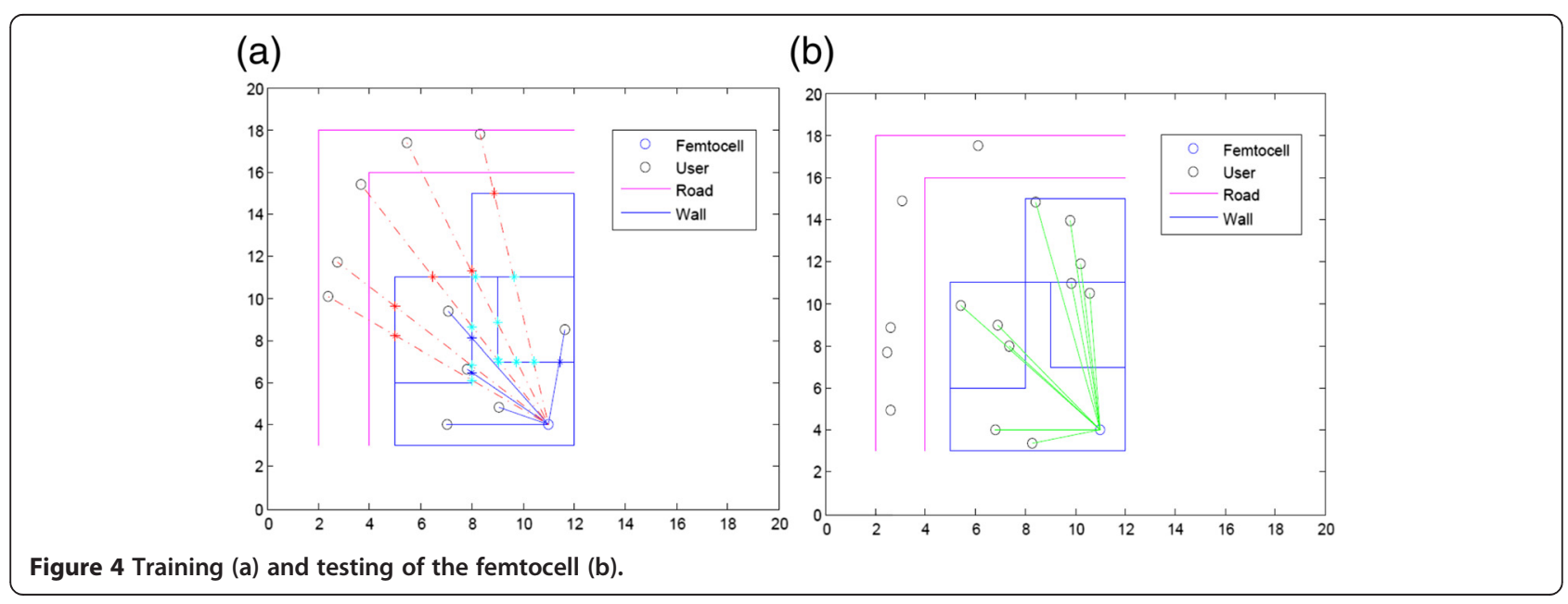




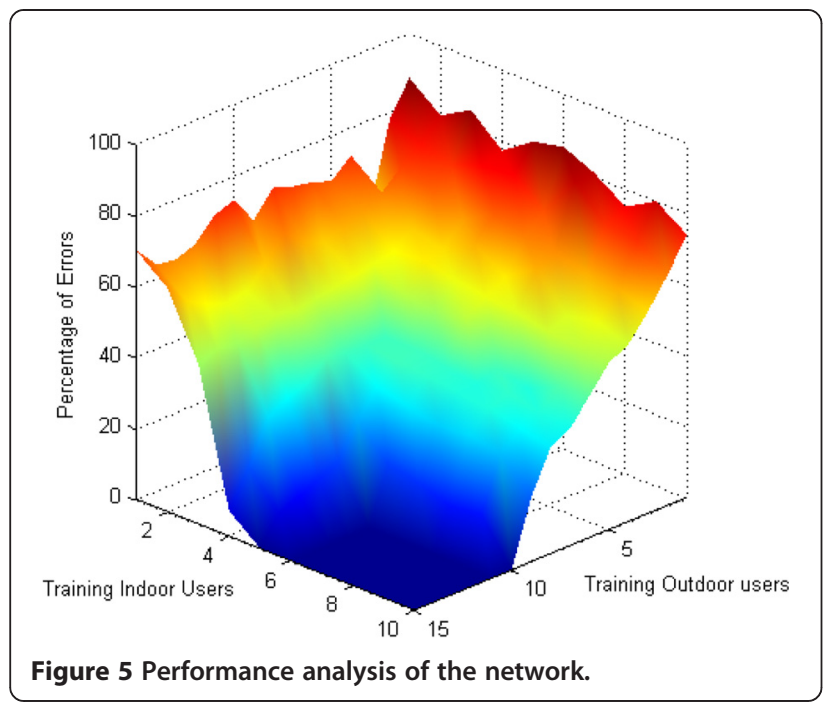

in the detection process with less number of training samples.

\section{Simulation and results}

To analyze the performance of user classification technique, a simulation environment is buildup in MATLAB ${ }^{\text {ma }}$ with a two-dimensional layout of a typical urban residence. A femtocell is placed in a corner of the house with a four-element $(M=4)$ antenna facing $90^{\circ}$ apart from each other on the same plane. In Figure 4a,b, a sample layout of the training and testing process is shown, respectively. The user equipment transmits omnidirectionally at $30 \mathrm{~dB}$ with no transmitting antenna gain. At the receiving end, all four antennas have different antenna gains for a particular user equipment. This reflects on the power received by each antenna.
The simulations are event-based and according to 3GPP standards. The plotted values are an average of 1,000 independent simulations. For the duration of the snapshot, all the users are assumed static so that the effects due to Doppler spread are neglected. The system parameters are given in Table 1. In Figure 5, the antennas are trained using 1 to 15 outdoor and 1 to 10 indoor users and in each step; the performance of the system is analyzed using 15 users randomly placed within the target area. All the users are assumed to be in the same elevation angle. The performance of the femtocell is illustrated varying the amount of training data. It clearly shows that the system benefits from the increased amount of training data. Further, randomly placed 15 samples each for indoor and outdoor areas is enough to make the system practically error-free. The figure is generated after training the femtocell with number of users shown next to the axes below. The initial stage with low outdoor users drags more errors in the estimation of users than the indoor users. It is because the outdoor users has more variation in their received signal power pattern than the indoors. However, after a certain number of training samples, the variation decreases and ends up in the error-free regions. Again, for the indoor users, it requires less number of user samples.

Figure 6a shows the mean square rate (MSE) of the of the ANN training process with 15 indoor and 15 outdoor users. Due to higher number of sample data points, the accuracy of the regular ANN training is more precise. In Figure $6 \mathrm{~b}$, the validation check shows a good fitness. However, the training process is always dependent on the house geometry and the indoor situation of the house. Furniture, walls, and electronic equipment are more of a concern in the indoor environment.
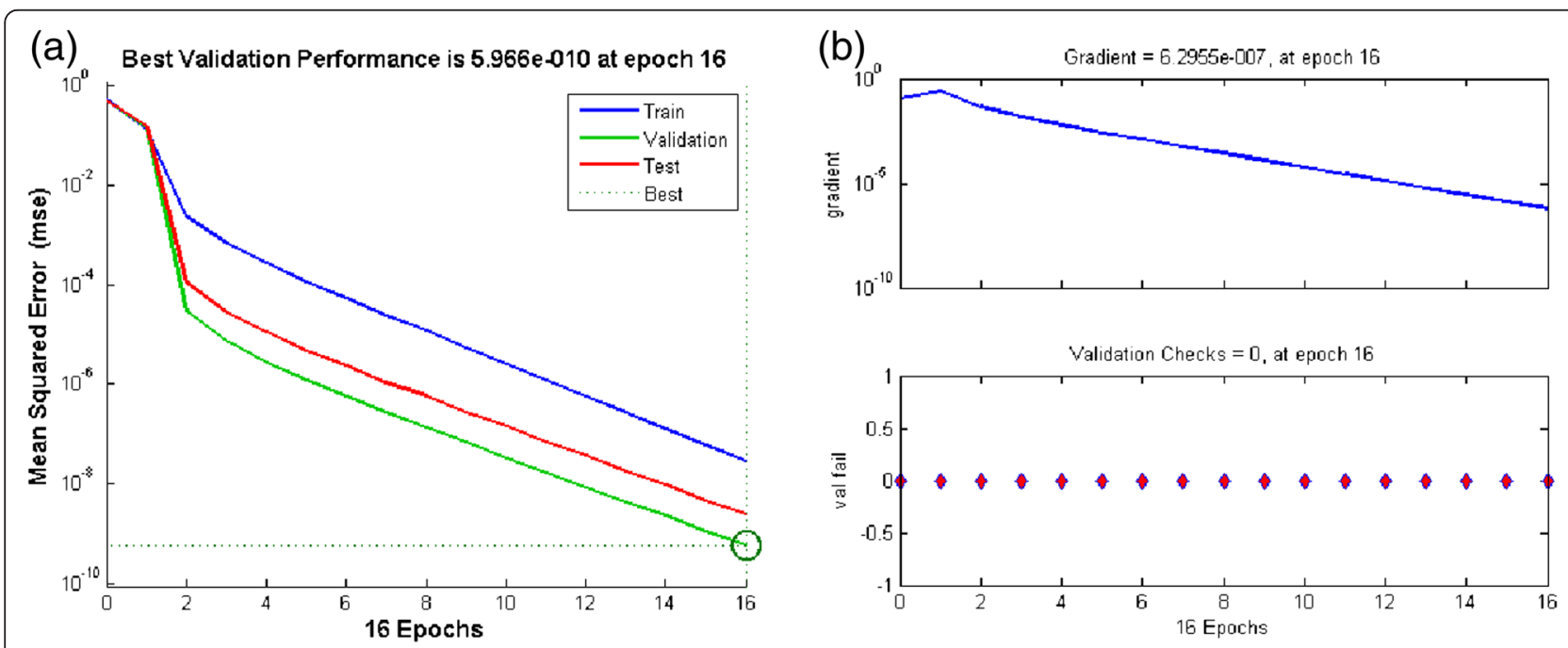

Figure 6 Best validation performance of ANN (a) and training state of ANN (b). 


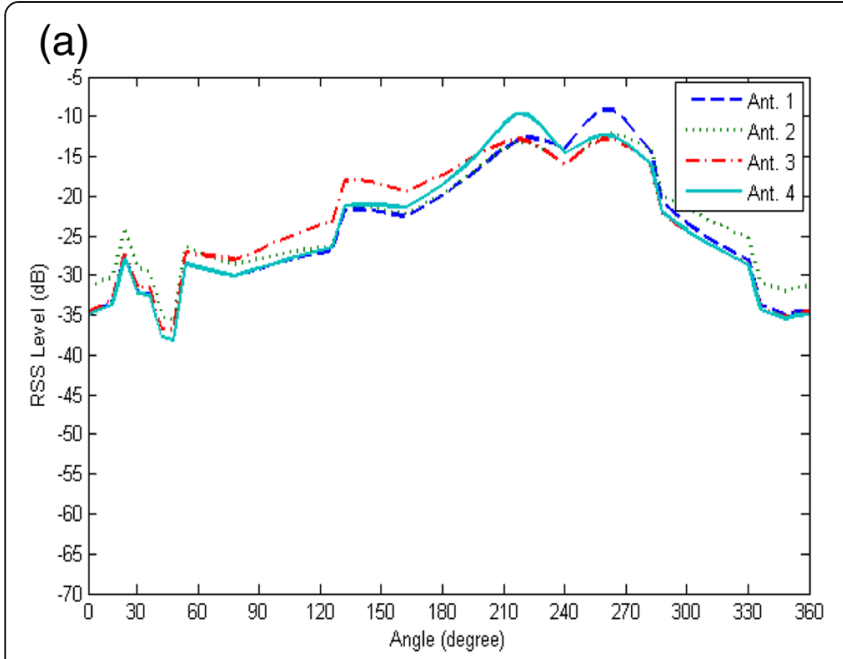

(b)

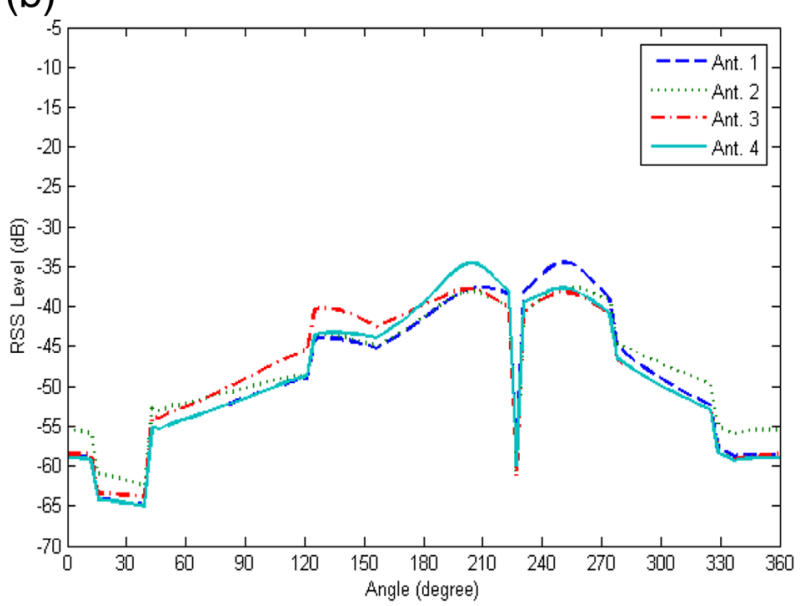

Figure 7 RSS level of the sample indoor users and outdoor users $(a, b)$.

A set of training sample is generated with one indoor and one outdoor users for each angle within the house and outside of the house. The angle is taken from $0^{\circ}$ to $360^{\circ}$ with a $2^{\circ}$ angular separation. The two indoor users are taken with a fair distance from each other to consider the effect of indoor walls and indoor shadowing. The power level reading of one set of indoor and outdoor users is plotted for a four-element antenna configuration. Figure $7 \mathrm{a}, \mathrm{b}$ shows the power level of indoor and outdoor users, respectively. Both users possess a multiple pattern of power reading for each angle. The difference of level depends on the directive pattern of the used sensing element, here, the microstrip antenna. The line graphs of the indoor and the outdoor curves have identical natures. The variation of the angle for both users follows a particular pattern instead of having noise, wall loss, and shadowing effects.

This study shows that the reconfigurable coverage area may be achieved by using simple training data that may be generated in real time if necessary. The large single lobe radiation pattern of the antenna with precisely desirable field of view allows the femtocell to operate efficiently. This approach can be applied to any antenna fulfilling the above criteria. This flexible approach to femtocell design is not limited in the area of coverage or total power of transmission. This concept is entirely scalable in terms of the area of coverage. This adaptive technique also serves as an added layer of security over existing wireless systems. Further, it requires very little computation and memory. Thus, it is ideal for implementation in wireless routers, femtocells, or any form of selective wireless broadcasting system.

\section{Conclusions}

The femtocell will occupy the major role in providing high indoor coverage in future cellular communication network. Closed access mechanism restricts the unwanted users to connect to primary users' personal femtocell. A cognitive closed access femtocell operation is presented here using neural network for a multi-element antenna femtocell. The performance of the system in recognizing the environment improves in keeping with the initial training values. The femtocell is trained by a set of trusted primary users. The training will remain valid as long as the femtocell's position and coverage area are not altered. Thus, any such femtocell will cover any arbitrary region. In the future work, the process will be developed for better femtocell performance with less training illustration.

\section{Competing interests}

The authors declare that they have no competing interests.

\section{Authors' contributions}

NM and MJS have carried out the antenna specification part, while MTI and $\mathrm{Ml}$ constructed the main concept of the article. TBAR and AUA have developed the coding, accumulated others work, and wrote down the manuscript. All authors read and approved the final manuscript.

\section{Acknowledgements}

The research was supported by Ministry of Education (MOE), Malaysia, under the grant scheme no. FRGSTOPDOWN/2014/TK03/UKM/01/1 and ICT fellowship grant for higher education and research in Information and Communication Technology under the Ministry of Post, Telecommunication and Information Technology, Bangladesh.

Received: 19 May 2014 Accepted: 2 March 2015

Published online: 28 March 2015

\section{References}

1. G De La Roche, A Valcarce, D López-Pérez, J Zhang, Access control mechanisms for femtocells. IEEE Commun. Mag. 48, 33-39 (2010) 
2. AU Ahmed, MT Islam, M Ismail, A Review on femtocell and its diverse interference mitigation techniques in heterogeneous network. Wireless Pers. Commun. 78, 85-105 (2014)

3. X Chu, Y Wu, L Benmesbah, W-K Ling, Resource allocation in hybrid macro/ femto networks, in 2010 IEEE Wireless Communications and Networking Conference Workshops (WCNCW), 2010, p. 1-5

4. AU Ahmed, MT Islam, M Ismail, M Ghanbarisabagh, Dynamic resource allocation in hybrid access femtocell network. Sci. World J. 2014, 7 (2014)

5. H Claussen, F Pivit, Femtocell coverage optimization using switched multi-element antennas, in IEEE International Conference on Communications, ICC'09, 2009, p. 1-6

6. H Claussen, F Pivit, LT Ho, Self-optimization of femtocell coverage to minimize the increase in core network mobility signalling. Bell Labs Tech. J. 14, 155-183 (2009)

7. ZD Zaharis, C Skeberis, TD Xenos, PI Lazaridis, J Cosmas, Design of a novel antenna array beamformer using neural networks trained by modified adaptive dispersion invasive weed optimization based data. IEEE Trans Broadcast. 59, 455-460 (2013)

8. TG Basha, P Sridevi, MG Prasad, Beam forming in smart antenna with precise direction of arrival estimation using improved MUSIC. Wireless Pers. Commun. 71(2), 1353-1356 (2012)

9. M Agatonović, Z Stanković, N Dončov, L Sit, B Milovanović, T Zwick, Application of artificial neural networks for efficient high-resolution 2D DOA estimation. Radioengineering 21, 1179 (2012)

10. D Inserra, AM Tonello, A multiple antenna wireless testbed for the validation of DoA estimation algorithms. AEU Int. J. Electron. Commun. 68, 10-18 (2014)

11. N Fonseca, M Coudyser, J-J Laurin, J-J Brault, On the design of a compact neural network-based DOA estimation system. IEEE Trans. Antennas Propag. 58, 357-366 (2010)

12. J Dudczyk, A Kawalec, Adaptive forming of the beam pattern of microstrip antenna with the use of an artificial neural network. Int. J. Antennas Propag. 2012, Article ID 935073, 13 pages (2012). doi:10.1155/2012/935073

13. T Ghouse Basha, A George, B Rajakumar, G Prasad, P Sridevi, A constructive smart antenna beam-forming technique with spatial diversity. IET Microw. Antennas Propag. 6, 773-780 (2012)

14. TG Basha, MG Prasad, P Sridevi, Hybrid technique for beam forming in smart antenna with spatial diversity. Int. J. Wirel. Mob. Comput. 5, 126-136 (2012)

15. AU Ahmed, MT Islam, R Azim, M Ismail, MF Mansor, Microstrip antenna design for femtocell coverage optimization. Int. J. Antennas Propag. 2014, 8 (2014)

16. AH El Zooghby, CG Christodoulou, M Georgiopoulos, A neural network-based smart antenna for multiple source tracking. IEEE Trans. Antennas Propag. 48, 768-776 (2000)

17. S Caylar, G Dural, K Leblebicioglu, Neural network method for direction of arrival estimation with uniform cylindrical microstrip patch array. IET Microw. Antennas Propag. 4, 153-161 (2010)

\section{Submit your manuscript to a SpringerOpen ${ }^{\circ}$ journal and benefit from:}

- Convenient online submission

- Rigorous peer review

- Immediate publication on acceptance

- Open access: articles freely available online

- High visibility within the field

- Retaining the copyright to your article

Submit your next manuscript at $>$ springeropen.com 